\title{
A TRIANGULAR DIAGRAM
}

\section{BY WILDER D. BANCROFT}

It is a very simple matter to express graphically the changes in composition of a given phase with the temperature when there are only two components; but a concentration-temperature diagram presents great difficulties when the number of components equals three. The problem has been solved in quite a number of ways as has been pointed out by Roozeboom. ${ }^{1}$ Most of the methods give a solid figure, the temperature being taken as the vertical axis ; but it is possible to tell a great deal from the projections of the curves for the monovariant systems upon a plane, even though the temperature can no longer be read directly. Schreinemakers ${ }^{2}$ takes for the $X$ and $\mathrm{Y}$ axes the amounts of two of the components in a constant quantity of the third. This is open to the objection that there is no place in the diagram for an anhydrous double salt, nor for solutions containing very little of the third component. Meyerhoffer ${ }^{8}$ has invented a diagram which has the merit of allowing one to take the temperature as one of the co-ordinates. In a system composed of tro salts and water, he measures the ratio of one salt to the other along one axis and the temperature along the other. This is serviceable in certain cases ; but is very limited in application since it neglects the relative quantities of both salts in respect to the third component. The method proposed by van Rijn van Alkemade ${ }^{4}$ seems to have no advantage over the diagram of Schreinemakers. Gibbs ${ }^{5}$ has suggested the use of a triangular diagram, the sum of the components being kept constant. If we take an equilateral triangle of unit height, the corners of the triangle will represent the pure components and any point within the triangle will represent some definite mixture of the three substances. The amount of each component

'Zeit. phys. Chem. 12, 367 ( I893). 'Ibid. 9, 67 ( I 892 ). 3Ibid. 5, 97 ( I890).
4Ibid. I , 306 ( I893):

5Trans. Conn. Acad. 3, I76 (I876). 
is given by the length of the perpendicular from the point to the side opposite the vertex corresponding to that component. This diagram has been used by Thurston ${ }^{1}$ in some work on alloys and was also suggested independently by Stokes ${ }^{2}$. Roozeboom ${ }^{3}$ has used a modification of this diagram. He takes the isosceles right-angle triangle, the equal sides being of unit length. The advantage of this arrangement is that one can use the ordinary co-ordinate paper; but it is open to the objection that there is a different scale along the hypotenuse from that along the sides, so that one of the components seems to occupy an exceptional position. While this is not serious in the case of two salts and water where the water is solvent and the salts solutes, it is a disadvantage in the ternary systems in which no such distinction exists and becomes impossible when the system of three components is considered as a subdivision of one containing four.

Roozeboom ${ }^{4}$ has proposed another form of triangular diagram which is distinctly superior to either of those already consideref. It consists of an equilateral triangle with lines ruled parallel to each side instead of perpendicular to them. The length of one side is taken equal to unity and the same scale is used for the binary systems in the sides of the triangle as for the ternary systems in the interior. It has seemed to me worth while to act upon this suggestion of Roozeboom and I have had made triangular co-ordinate paper with lines ruled parallel to the three sides. Since there are some interesting geometrical relations connected with the use of this diagram, only a few of which have been mentioned by Roozeboom, I propose to give a brief sketch of the information which can be read off directly from Figure I.

We will suppose that we have the components, A, B and $C$ and that the sum of these components always equals Ioo. The choice of units is entirely arbitrary but must be adhered to when made. The corners of the diagram marked $A, B$ and $C$ represent phases

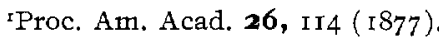

${ }^{2}$ Proc. Roy. Soc. 49, I74 (I89I).

3Zeit. phys. Chem. 12, 369, (1893).

4Zeit. phys. Chem. 15, I43 (1894). 
containing one hundred percent of the components $A, B$ and $C$ respectively. The composition of any phase containing $\mathrm{A}$ and $\mathrm{B}$ only will be represented by a point on the line $A B$; while phases containing only $A$ and $C$ or only $B$ and $C$ will be found on the lines $\mathrm{AC}$ and $\mathrm{BC}$ respectively. Any phase containing all three components will be represented by some point in the interior of the diagram. The composition corresponding to the point $O$ can be read off in a

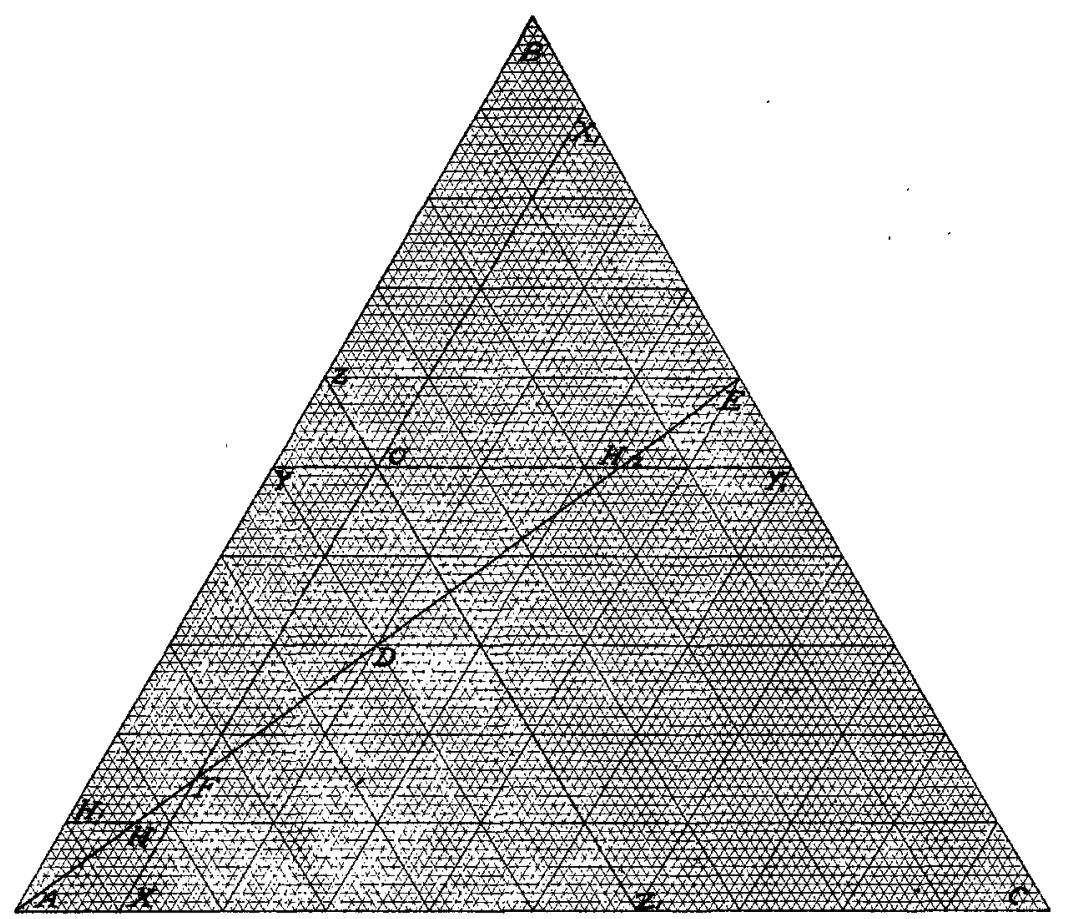

FIG I.

number of different ways. Through $O$ draw lines parallel to each of the three sides. The usual way of determining the composition is to start from one corner as the origin and measure along the two sides. Thus AX measures the amount of $\mathrm{C}, \mathrm{AY}$ the amount of $\mathrm{B}$ in the unit mass of the phase, while the amount of $A$ is given by the difference. In this particular case the co-ordinates of the point $\mathrm{O}$ are $\mathrm{A}=40, \mathrm{~B}=5^{\circ}, \mathrm{C}=$ ro. Sometimes it is desirable to read 
off the value from one side only, let us say the side $A B . A Y$ is a measure of $\mathrm{B}, \mathrm{YZ}$ of $\mathrm{C}$ and $\mathrm{BZ}$ of $\mathrm{A}$. This will easily be seen to be true for $\mathrm{OY}=\mathrm{YZ}$ by construction, being sides of an equilateral triangle. $\mathrm{AX}=\mathrm{YO}$ since they are opposite sides of a parallelogram. $B Z$ is the remainder of the line $A B$ and therefore is a measure of the quantity of $A$ in the unit mass of the phase. The same process can be carried out for all three sides of the diagram if desired. The lines $\mathrm{BZ}, \mathrm{OX}_{1}, \mathrm{OY}_{1}, \mathrm{X}_{1} \mathrm{Y}_{1}$ and $\mathrm{CZ}_{1}$ are all equal and measure $\mathrm{A}$; the lines $\mathrm{AY}, \mathrm{OX}, \mathrm{OZ}_{1}, \mathrm{XZ}_{1}$ and $\mathrm{CY}_{1}$ are all measures of the component $\mathrm{B}$ while $\mathrm{AX}, \mathrm{OY}, \mathrm{OZ}, \mathrm{Y} . \mathrm{Z}$ and $\mathrm{BX}$, perform the same function for $C$. This may be summed up into the following general rule for determining parts of each component in the mixture: Lines being extended from $O$ parallel to the sides, the interior sect gives the value for the opposite component and each external sect that for the component at the opposite end. It may not be amiss to mention that the concentration $\mathrm{A}=40, \mathrm{~B}=5 \circ, \mathrm{C}=10$, would be represented by the same point $O$ if the co-ordinate paper were made with lines perpendicular to the sides instead of parallel to them.

Besides knowing the amounts of $A, B$ and $C$ in one hundred parts of $\mathrm{A}+\mathrm{B}+\mathrm{C}$, it is sometimes desirable to determine the quantity of $\mathrm{B}$ in one hundred parts of $\mathrm{B}+\mathrm{C}$, for instance. This is also given by the diagram. Let us suppose a phase having the composition $\mathrm{A}=5 \mathrm{O}, \mathrm{B}=3 \mathrm{O}, \mathrm{C}=20$. This phase will be represented by the point $D$. Draw a line from the corner $A$ through $D$. The line $\mathrm{AD}$ cuts $\mathrm{BC}$ at $\mathrm{E}$. The distance $\mathrm{CE}$ gives the amount of $\mathrm{B}$ in one hundred parts of $\mathrm{B}+\mathrm{C}$; in this case $\mathrm{CE}=60 . \mathrm{BE}$ will therefore give the anount of $C$ in one hundred parts of $B+C$. The line $\mathrm{AD}$ cuts the line denoting a Io percent concentration of $\mathrm{B}$ at $\mathrm{H}$ and ten times the distance $\mathrm{HH}_{1}$ or 66.7 is the amount of $\mathrm{C}$ in one hundred parts of $B$. In the same way ten times the distance $F X$ gives the parts of $\mathrm{B}$ in one hundred parts of $\mathrm{C}$. If the multiplication of the distance by ten seems to introduce too large an error, one can take the point at which the line AD cuts the line for 20 percent or 50 percent concentration of one component and then the distance is multiplied by five or two respectively. Thus twice $\mathrm{H}_{2} \mathrm{X}$ is equal to ten times $\mathrm{HH}_{1}$ and gives the parts of $\mathrm{C}$ in one hundred parts of $\mathrm{B}$. The line for $5^{\circ}$ percent of $\mathrm{C}$ in the ternary solution cuts $\mathrm{AD}$ outside the 
diagram. To determine the corresponding relation between $A$ and $B$ or $A$ and $C$ we have only to draw the line $C D$ or $B D$ and proceed as before.

If one wish to present simultaneotusly the behavior of three components, or any part of them or each one separately this can be done by a very slight modification of the diagram. In Fig. 2 are given what few data there are for the system made up of potassium, sodium

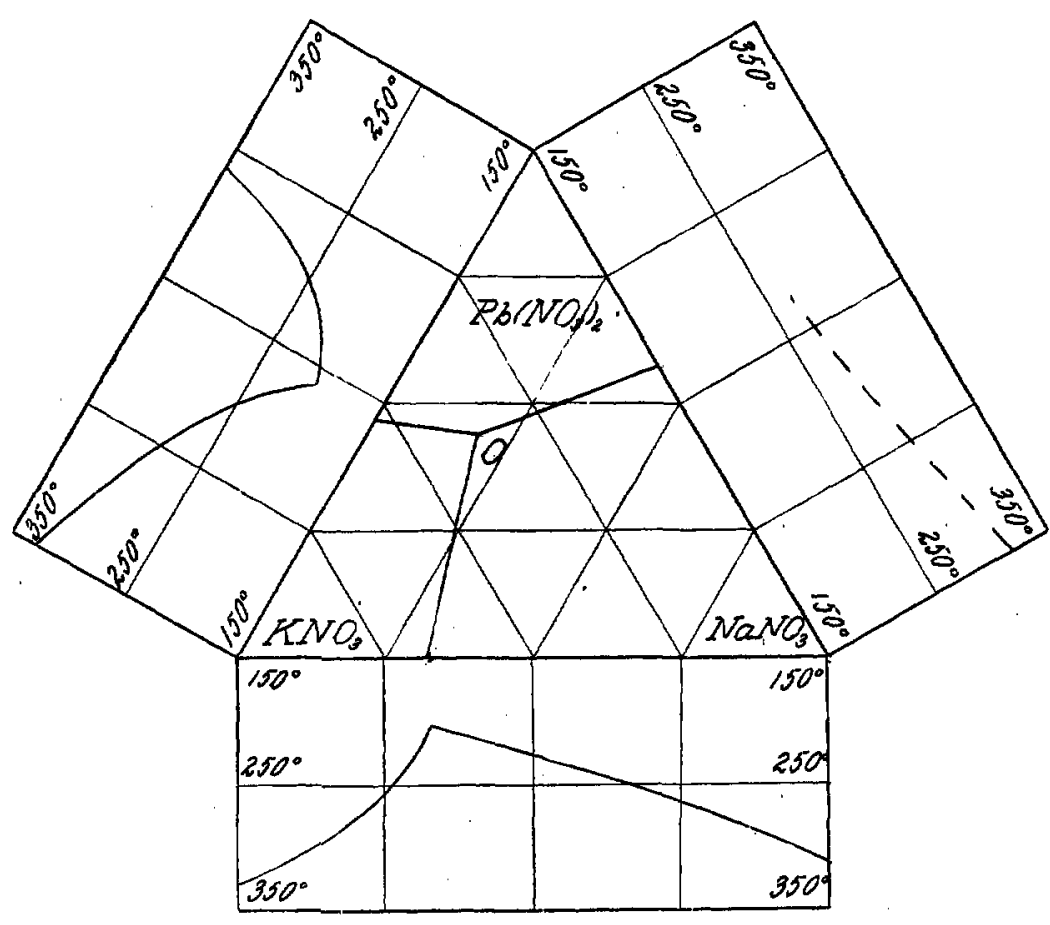

FiG. 2.

and lead nitrates. ${ }^{1}$ In the central triangular diagram the lines meeting at $O$ represent the three monovariant systems containing two solid phases, solution and vapor. Perpendicular to each side of the triangle are the temperature axes and the curves which are given there are the concentration-temperature diagrams for the three binary systems. The curves for potassium and lead nitrates do not reach

${ }^{ \pm}$Guthrie, Phil. Mag. [5] 17, 472 (I884). 
the point corresponding to one hundred percent of lead nitrate because this substance decomposes before its melting point is reached. Only two points are known for the system, lead and sodium nitrates, the melting point of sodium nitrate and the composition and melting point of the eutectic alloy. For this reason only one branch of the curve is given and that in a dotted line. It would be possible to have the temperature axes follow the lines of the co-ordinate paper, making angles of sixty and one hundred and twenty degrees with the sides of the triangle. The objection to this is that one branch of the curve for the binary system is crowded together while the other is drawn out giving a very distorted effect. In each corner of the figure it is possible to draw a line perpendicular to one of the temperature axes and present the pressure-temperature diagram for each of the single components. This has not been done because the vapor pressures of the three salts have never been determined.

Returning to the concentration-temperature diagrans for the different binary systems, there are one or. two points yet to be considered. The curves in these diagrams represent graphically the temperatures of the points along the sides of the central triangle. If we wish to learn the temperature of any point on any side of the inner triangle we have only to erect a perpendicular from the point to the concentration-temperature curve for the binary system and read off the temperature at the point of intersection. This can best be seen perhaps if we consider the complete diagran for a ternary system. As has already been stated, this forms a solid figure with the temperature as vertical axis. If now we imagine the three concentration-temperature diagrams for the binary systems revolved ninety degrees on the sides of the central triangle as axes we shall have the shell of the solid figure just referred to and the curves in the rotated diagrams are the outside upper edges of the complete model for a ternary system. The method of representation adopted here shows the temperatures of points along the sides of the triangle upon which the actual model is projected, but it does not give any information as yet in regard to the temperatures in the interior of the triangle. The usual way of doing this is to draw isothermal lines; but this obscures the diagram by multiplying the number of lines. In many cases it will therefore be of advantage to represent 
these temperatures in the side diagrams of the binary systems. This can be done by a series of lines each giving the relation between temperature and concentration when two components vary and the third remains constant. The curves already drawn in the side diagrams are for one hundred parts of two of the components and zero parts of the third. The succeeding curves would represent systems containing one hundred parts of two components and ten, twenty, thirty, etc., parts of the third substance. To determine what concentration this refers to in the triangular diagram where the sum of the three components is always one hundred, it is only necessary to drop a perpendicular to the side of the triangle and draw a line from there to the opposite vertex. The point at which this line cuts the line for ten, twenty or thirty percent concentration of the third component represents the desired composition.

While it is not possible to represent the compositions and temperatures of any phase in a ternary system by single points in a plane, it is possible to do this if one uses two points for each determination. This method has been employed by van der Heide in his study of the system, potassium, and magnesium sulfates, and water. $\mathrm{He}$ expresses the concentrations in parts of each component per hundred parts of the solution. The temperature is taken as one axis while the concentration of the two salts are laid off on the other axis right and left from a zero point. Under these circumstances there are are two points for each nonvariant system and two curves for each monovariant system. This method has very great advantages. The temperature corresponding to each concentration is given directly and one can determine from the diagram the accuracy or inaccuracy of Meyerhoffer's theorem that the sudden change of direction of the solubility curve in passing through the inversion temperature does not affect the solubility of the solid phase which remains. In addition to the behavior of the binary systems, that of each of the salts with water can easily be shown, as indeed van der Heide has pointed ont. The objection to be made to the diagram in its present form is that the concentration of the third component, water, can not be measured directly; but has to be obtained by a process of addition and subtraction and also that solutions contain-

'Zeit. phys. Chem. 12, 416 (1893). 
ing only two salts can not be represented by a single line. This could be avoided by a very slight modification, measuring the temperature along the ordinates. Let the abscissas be a line of definite length corresponding to one hundred parts of the solution. Lay off the concentration of one component from the extreme left of this line and the concentration of the second from the extreme right. The concentration of the third component is given by the distance between these points. The binary systems can then be represented in the same diagram by single lines since one of the lines for the ternary system coincides with one of the $Y$ axes when either the first or second component disappears while the two lines coalesce if it is the third component which vanishes.

Cornell University. 